\title{
ROBUST ADAPTIVE TRACKING CONTROL FOR A CLASS OF PERTURBED UNCERTAIN NONLINEAR SYSTEMS
}

\author{
Tieshan Li, ${ }^{1}$ Yansheng Yang, Biguang Hong, \\ and Junshen Ren \\ Navigation College, Dalian Maritime University, Dalian \\ 116026, China
}

\begin{abstract}
A novel robust adaptive controller is presented for a wide class of strict feedback uncertain nonlinear system with unknown virtual control coefficients under bounded exogenous disturbances. Combined Nussbaum gain with the backstepping technique, the proposed design algorithm, which does not require a priori knowledge of the signs of the unknown virtual control coefficients, is proved to be able to guarantee the resulting closed-loop system globally uniformly ultimately bounded (GUUB). Moreover, the output of the system is proven to converge to a small neighborhood of the origin. Simulation results are presented to validate the effectiveness of the proposed controller. Copyright@ 2005 IFAC.
\end{abstract}

Keywords: Uncertain nonlinear systems, robust adaptive control, backstepping design, virtual control gain function, Nussbaum type gain.

\section{INTRODUCTION}

During the past few years, a lot of researches and significant progress have been made in the adaptive control of nonlinear systems via feedback linearization (Sastry and Isidori, 1989), (Kanellakopoulos et al., 1991), which has evolved as a powerful methodology. For a class of nonlinear systems transformable to a parametric strictfeedback canonical ones, a recursive design procedure, adaptive backstepping approach, has been presented (Isidori, 1995), (Krstic et al., 1995), (Marino and Toper, 1993). The overparametrization problem was soon eliminated via introducing the concept of tuning function (Krstic et al., 1992). Recently, nonlinear damping was also introduced in the controller to improve transient

\footnotetext{
1 This work was supported in part by the research fund for the Doctoral Program of Higher Education under Grant no.(20020151005) and National Natural Science Fund (60474014)
}

performance (Kanellakopoulos, 1993). However, all these adaptive controllers deal with the case of parametric uncertainties only, seldom with the uncertainties on modeling and external disturbances, nor with the case including unknown virtual control gain functions.

When there is no a priori knowledge about the signs of virtual control coefficients, adaptive control of such systems becomes much more difficult. The first solution was given in (Nussbaum, 1983) for a class of first-order linear systems, where the Nussbaum type gain was originally proposed. Without the requirement for monotone increasing arguments for the Nussbaum functions, the same technique has been extended to higher order systems for constant virtual control coefficients in (Ye and Jiang, 1998), (Ge and Wang, 2002), using decoupled backstepping. Recently, with respect to both unknown virtual control gain function and unknown time delay systems, two adaptive neural controllers are presented for a class of strict- 
feedback nonlinear systems in (Ge et al., 2003) using the same technique and integral Lyapunov functions.

Motivated by the works (Ye and Jiang, 1998; Ge and Wang, 2002), and based on the Nussbaumtype gain and the decoupled backstepping techniques, a novel robust adaptive tracking controller is presented for a wide class of perturbed uncertain nonlinear systems with unknown virtual control gain functions, which are complex than the systems in (Ye and Jiang, 1998), (Ge and Wang, 2002), and without the help of integral Lyapunov functions, the design procedure is simple than that in (Ge et al., 2003), such that the closed-loop system is globally uniformly ultimately bounded (GUUB), and additionally, the tracking error is proven to be able to converge to a small neighborhood of the origin as small as desired by an appropriate choice of the design parameters in the controller. In a word, the main contribution is that, this paper enlarges the class of perturbed strict-feedback uncertain nonlinear systems for which global robust adaptive tracking control can be designed.

\section{PROBLEM FORMULATION AND PRELIMINARIES}

In this paper, we treat the control problem of nonlinear systems transformable to the following strict-feedback form

$$
\left\{\begin{array}{l}
\dot{x}_{i}=g_{i}\left(\bar{x}_{i}\right) x_{i+1}+\theta_{i}^{T} \psi_{i}\left(\bar{x}_{i}\right)+\Delta_{i}(t, x), \\
\quad 1 \leq i \leq n-1 \\
\dot{x}_{n}=g_{n}(x) u+\theta_{n}^{T} \psi_{n}(x)+\Delta_{n}(t, x) \\
y=x_{1}
\end{array}\right.
$$

where $\bar{x}_{i}=\left[x_{1}, \ldots, x_{i}\right]^{T}, x=\left(x_{1}, \ldots, x_{n}\right) \in R^{n}$ , $u \in R, y \in R$ are the state variables, system input and output respectively, $\theta_{i} \in R^{p}$ denotes the unknown constant parameter vector, $\psi_{i}\left(x_{1}, \ldots, x_{i}\right)$ is known smooth function, $g_{i}$ is unknown virtual control gain function, and its sign is also unknown, $\Delta_{i}(t, x)$ is the bounded uncertainties, which can include modeling uncertainties, external disturbances, etc. The control goal is to design a robust adaptive tracking controller such that the output $y(t)$ can follow a desired reference signal $y_{d}(t)$, while all the signals and states involved are GUUB.

Assumption 1 : There exist constants $g_{i 0}$ and known smooth functions $\bar{g}_{i}\left(\bar{x}_{i}\right)$ such that $0<$ $g_{i 0} \leq\left|g_{i}\left(\bar{x}_{i}\right)\right| \leq \bar{g}_{i}\left(\bar{x}_{i}\right), \forall \bar{x}_{i} \in R^{i}$. In addition, $\bar{g}_{i}\left(\bar{x}_{i}\right)$ take value in the unknown closed intervals $I_{i}:=\left[l_{i}^{-}, l_{i}^{+}\right] \subset\left[g_{i 0},+\infty\right)$.

Assumption 2 : There exists an completely unknown constant $b_{i}$ and known smooth function $\psi_{1, i}$, such that

$$
g_{i}\left(\bar{x}_{i}\right)=b_{i} \psi_{1, i}\left(\bar{x}_{i}\right)
$$

Remark 1: In the works of (Ye and Jiang, 1998),(Ge and Wang, 2002), $g_{i}$ 's are constants, and (Ye and Jiang, 1998) did not consider the perturbed uncertainties. So those systems are one special case of the systems in this paper.

Assumption 3: There exists an unknown positive constant $\lambda_{i}$, for all $(t, x) \in R^{+} \times R^{n}$,

$$
\left|\Delta_{i}(t, x)\right| \leq \lambda_{i} \phi_{i}\left(\bar{x}_{i}\right)
$$

where $\phi_{i}$ is a known nonnegative smooth function.

Assumption 4: The reference signal $y_{d}(t)$ has up to its $n$th time derivative, and $Y_{d}=$ $\left[y_{d}, \dot{y}_{d}, \ldots, y_{d}^{(n)}\right]^{T}$ is bounded.

Now we introduce a useful Lemma on Nussbaumtype function gain (Nussbaum, 1983) which will be used throughout this paper (For clarity, an even Nussbaum function $N(\kappa)=\exp \left(\kappa^{2}\right) \cos \left(\frac{\pi \kappa}{2}\right)$ is used throughout this paper.).

Lemma 1:(Ge et al., 2003) Let $V(\cdot)$ and $\kappa(\cdot)$ be smooth functions defined on $\left[0, t_{f}\right)$ with $V(t) \geq 0$ $, \forall t \in\left[0, t_{f}\right), N(\cdot)$ be an even smooth Nussbaumtype function. If the following inequality holds:

$$
\begin{aligned}
V(t) \leq C_{0}+e^{-c_{1} t} \int_{0}^{t} g(x(\tau)) N(\kappa) \dot{\kappa} e^{c_{1} t} d \tau \\
+e^{-c_{1} t} \int_{0}^{t} \dot{\kappa} e^{c_{1} t} d \tau, \forall t \in\left[0, t_{f}\right)
\end{aligned}
$$

where $c_{1}>0, g(x(\tau))$ is a time-varying function which takes values in the unknown closed interval $I:=\left[l^{-}, l^{+}\right]$with $0 \notin I$, and $C_{0}$ represents some suitable constant, then $V(t), \kappa(t)$ and $\int_{0}^{t}(g(x(\tau)) N(\kappa(\tau))+1) \dot{\kappa}(\tau) d \tau$ must be bounded in $\left[0, t_{f}\right)$.

Remark 2: According to (Ryan, 1991), if the solutions to the closed-loop system exist, then $t_{f}=\infty$. So the boundedness result in Lemma 1 is able to be extended to globally uniformly ultimately bounded(GUUB).

\section{DESIGN PROCEDURE AND STABILITY ANALYSIS}

For simplification and conciseness, we define Lyapunov candidate function $V_{i}$, for $i=1, \ldots, n$, as follows

$$
V_{i}=\frac{1}{2} z_{i}^{2}+\frac{1}{2} \tilde{\Theta}_{i}^{T} \Gamma_{i}^{-1} \tilde{\Theta}_{i}+\frac{1}{2 \gamma_{i}} \tilde{\lambda}_{i}^{* 2}
$$

where $\gamma_{i}>0, \Gamma_{i}$ is positive definite matrix to be design later. define estimation errors $(\tilde{*})=(\cdot)-(\hat{*})$

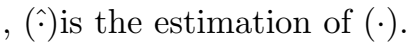

Theorem 1: Consider the closed-loop adaptive system consisting of the system (1) under As- 
sumptions 1-4, the robust adaptive tracking control law (6) and the intermediate stabilizer (7) and adaptation laws (10) and (11), for $i=i, \ldots, n$,

$$
\begin{gathered}
u=\alpha_{n} \\
\alpha_{i}=N\left(\kappa_{i}\right) \xi_{i} \\
\dot{\kappa}_{i}=\xi_{i} z_{i} \\
\xi_{i}=c_{i} z_{i}+\hat{\Theta}_{i}^{T} \Phi_{i}+\beta_{i}+\hat{\lambda}_{i}^{*} \bar{\phi}_{i} \tanh \left(\frac{z_{i} \bar{\phi}_{i}}{\varepsilon_{i}}\right) \\
\dot{\hat{\Theta}}_{i}=\Gamma_{i}\left[\Phi_{i} z_{i}-\sigma_{\theta i}\left(\hat{\Theta}_{i}-\dot{\hat{\Theta}}_{i}^{0}\right)\right] \\
\dot{\hat{\lambda}}_{i}^{*}=\gamma_{i} z_{i} \bar{\phi}_{i} \tanh \left(\frac{z_{i} \bar{\phi}_{i}}{\varepsilon_{i}}\right)-\gamma_{i} \sigma_{\lambda i}\left(\hat{\lambda}_{i}^{*}-\lambda_{i}^{0}\right)
\end{gathered}
$$

can guarantee the following properties: i) all the signals and solutions in the closed-loop system remain GUUB, ii) for any given $\varepsilon^{*}>\sqrt{\sum_{i=1}^{n} 2 \rho_{i}}$, there exists a $T>0$, such that $|z(t)| \leq \varepsilon^{*}$ for all $t \geq T$. Furthermore, $\varepsilon^{*}$ can be made as small as desired by an appropriate choice of the design parameters such that the tracking error $z_{1}=y(t)-y_{d}(t)$ satisfies the property of $\lim _{t \rightarrow \infty}\left|z_{1}(t)\right| \leq \varepsilon^{*}$. Correspondingly, the system output $y(t)$ satisfies

$$
|y(t)| \leq \sqrt{2\left(\rho_{1}+C_{1}\right)}+\left|y_{d}(t)\right| .
$$

where $c_{i}, \varepsilon_{i}, \sigma_{\theta i}, \sigma_{\lambda i}, \theta_{i}^{0}, \lambda_{i}^{0}$ are positive design parameters. And

$C_{1}=\sup \int_{0}^{t}\left(\left(g_{1} N\left(\kappa_{1}\right)+1\right) \dot{\kappa}_{1}+g_{1} z_{2}^{2}\right) e^{-c_{11}(t-\tau)} d \tau$.

Proof: The proof will be obtained via the following backstepping design procedure.

First, we define $\rho_{i}=\delta_{i} / c_{i i}$

$$
\begin{gathered}
\delta_{i}=\lambda_{i}^{*} \varepsilon_{i}+\frac{1}{2} \sigma_{\theta i}\left|\Theta_{i}-\Theta_{i}^{0}\right|^{2}+\frac{1}{2} \sigma_{\lambda i}\left|\lambda_{i}^{*}-\lambda_{i}^{0}\right|^{2}, \\
c_{i i}:=\min \left\{2\left(c_{i}-\frac{1}{4}\right), \frac{\sigma_{\theta i}}{\lambda_{\max }\left(\Gamma_{i}^{-1}\right)}, \sigma_{\lambda i} \gamma_{i}\right\} .
\end{gathered}
$$

Step 1: Let $z_{1}=x_{1}-y_{d}, z_{2}=x_{2}-\alpha_{1}$, then

$$
z_{1} \dot{z}_{1}=z_{1}\left[g_{1}\left(z_{2}+\alpha_{1}\right)+\theta_{1}^{T} \psi_{1}+\Delta_{1}-\dot{y}_{d}\right]
$$

Note that (3) and the Schwarz inequality, and let $i=1$ in (5), then the derivative of $V_{1}$ can be obtained

$$
\begin{aligned}
& \dot{V}_{1} \leq\left[g_{1}\left(x_{1}\right) z_{1} z_{2}+z_{1} g_{1}\left(x_{1}\right) \alpha_{1}+\Theta_{1}^{T} \Phi_{1}\left(x_{1}\right) z_{1}\right. \\
& \left.\quad-z_{1} \dot{y}_{d}\right]+\left|z_{1}\right| \lambda_{1} \bar{\phi}_{1}\left(x_{1}\right)-\tilde{\Theta}_{1}^{T} \Gamma^{-1} \dot{\hat{\Theta}}_{1}-\tilde{\lambda}_{1}^{T} \gamma_{1}^{-1} \dot{\hat{\lambda}}_{1}
\end{aligned}
$$

where $\Theta_{1}=\theta_{1}, \bar{\phi}_{1}=\phi_{1}, \Phi_{1}=\psi_{1}$.
By substituting (7)-(11), for $i=1$, into (16), then

$$
\begin{aligned}
\dot{V}_{1} \leq & g_{1}\left(\bar{x}_{1}\right) z_{1} z_{2}+g_{1}\left(\bar{x}_{1}\right) N\left(\kappa_{1}\right)\left(\dot{\kappa}_{1}\right)+\dot{\kappa}_{1} \\
& +\hat{\lambda}_{1}\left|z_{1}\right| \bar{\phi}_{1}-\hat{\lambda}_{1} z_{1} \bar{\phi}_{1}\left(x_{1}\right) \tanh \left(\frac{z_{1} \bar{\phi}_{1}\left(x_{1}\right)}{\varepsilon_{1}}\right) \\
& -c_{1} z_{1}^{2}+\sigma_{\theta 1} \tilde{\Theta}_{1}^{T}\left(\hat{\Theta}_{1}-\Theta_{1}^{0}\right)+\sigma_{\lambda 1} \tilde{\lambda}_{1}^{T}\left(\hat{\lambda}_{1}-\lambda_{1}^{0}\right) \\
\leq & -\left(c_{1}-\frac{1}{4}\right) z_{1}^{2}-\frac{1}{2} \sigma_{\theta 1}\left|\tilde{\Theta}_{1}\right|^{2}-\frac{1}{2} \sigma_{\lambda 1}\left|\tilde{\lambda}_{1}\right|^{2} \\
& +\left(g_{1} N\left(\kappa_{1}\right)+1\right) \dot{\kappa}_{1}+\delta_{1}+g_{1}^{2} z_{2}^{2} \\
\leq & -c_{11} V_{1}+\left(g_{1} N\left(\kappa_{1}\right)+1\right) \dot{\kappa}_{1}+\delta_{1}+g_{1}^{2} z_{2}^{2}
\end{aligned}
$$

In the above analysis, the Young's inequality $g_{1}\left(\bar{x}_{1}\right) z_{1} z_{2} \leq \frac{1}{4} z_{1}^{2}+g_{1}^{2}\left(\bar{x}_{1}\right) z_{2}^{2}$ and the facts $0 \leq|x|-x \tanh \left(\frac{x}{\varepsilon}\right) \leq 0.2785 \varepsilon, \varepsilon>0, x \in R$, $\tilde{\theta}^{T}\left(\hat{\theta}-\theta_{0}\right)=\frac{1}{2}|\tilde{\theta}|^{2}+\frac{1}{2}\left|\hat{\theta}-\theta_{0}\right|^{2}-\frac{1}{2}\left|\theta-\theta_{0}\right|^{2}$ are used.

Similar to (Ge and Wang, 2002)and (Ge et al., 2003), then

$$
\begin{aligned}
0 & \leq V_{1}(t) \\
& \leq \rho_{1}+V_{1}(0)+e^{-c_{11} t} \int_{0}^{t} g_{1} N\left(\kappa_{1}\right) \dot{\kappa}_{1} e^{c_{11} \tau} d \tau \\
& +e^{-c_{11} t} \int_{0}^{t} \dot{\kappa}_{1} e^{c_{11} \tau} d \tau+e^{-c_{11} t} \int_{0}^{t} g_{1}^{2} z_{2}^{2} e^{c_{11} \tau} d \tau
\end{aligned}
$$

From (18), if there is no extra term $e^{-c_{11} t} \int_{0}^{t} g_{1}^{2} z_{2}^{2} e^{c_{11} \tau} d \tau$ within the inequality, it can be concluded that $V_{1}(t), \kappa_{1}$, as well as $\alpha_{1}, \hat{\theta}_{1}$ are all GUUB by Lemma 1 and Remark 2. Due to the presence of the extra term in (18), Lemma 1 cannot be applied directly. However, if $z_{2}$ can be regulated as bounded, then the regulation of $z_{1}$ is achieved.

From Assumption 1, the following inequality can be obtained (Ge et al., 2003),

$$
e^{-c_{11} t} \int_{0}^{t} g_{1}^{2} z_{2}^{2} e^{c_{11} \tau} d \tau \leq \frac{1}{c_{11}} l_{1}^{+2} \sup _{\tau \in[0, t]} z_{2}^{2}(\tau)
$$

Thus, if $z_{2}$ is regulated as bounded, then the boundedness of the extra term can be readily concluded from (19). The effect of the extra term will be tackled in the following steps.

Step $i(2 \leq i \leq n-1)$ Similar procedures are taken recursively for each step of $i=2, \cdots, n-1$.

Define $z_{i}=x_{i}-\alpha_{i-1}$, then we can obtain

$$
\begin{aligned}
z_{i} \dot{z}_{i} & =z_{i}\left[g_{i}\left(z_{i+1}+\alpha_{i}\right)+\theta_{i}^{T} \psi+\Delta_{i}+\beta_{i}\right. \\
& \left.-\sum_{j=1}^{i-1} \frac{\partial \alpha_{i-1}}{\partial x_{j}}\left(b_{j} \psi_{1 j}\left(\bar{x}_{i}\right) x_{j+1}+\theta_{j}^{T} \psi_{j}+\Delta_{j}\right)\right]
\end{aligned}
$$

where

$$
\begin{aligned}
\beta_{i}= & -\sum_{j=1}^{i-1} \frac{\partial \alpha_{i-1}}{\partial \hat{\theta}_{j}} \dot{\hat{\theta}}_{j}-\sum_{j=1}^{i-1} \frac{\partial \alpha_{i-1}}{\partial \hat{\lambda}_{j}} \dot{\hat{\lambda}}_{j} \\
& -\sum_{j=0}^{i-1} \frac{\partial \alpha_{i-1}}{\partial y_{d}^{(j)}} y_{d}^{(j+1)}-\frac{\partial \alpha_{i-1}}{\partial \kappa_{i-1}} \dot{\kappa}_{i-1}
\end{aligned}
$$

From Assumption 3, the following inequality holds 


$$
z_{i}\left(\Delta_{i}-\sum_{j=1}^{i-1} \frac{\partial \alpha_{i-1}}{\partial x_{j}} \Delta_{j}\right) \leq \lambda_{i}^{*}\left|z_{i}\right| \bar{\phi}_{i}\left(\bar{x}_{i}\right)
$$

where $\lambda_{i}^{*}=\max \left\{\lambda_{1}, \ldots, \lambda_{i}\right\}$, and

$$
\bar{\phi}_{i}\left(\bar{x}_{i}\right)=\phi_{i}+\sum_{j=1}^{i-1}\left|\frac{\partial \alpha_{i-1}}{\partial x_{j}}\right| \phi_{j}
$$

From Assumption 2, define

$$
\begin{gathered}
\Theta_{i}=\left[b_{1}, \ldots, b_{i-1}, \theta_{i}^{T}, \theta_{1}^{T}, \ldots, \theta_{i-1}^{T}\right]^{T} \\
\Phi_{i}=\left[-\frac{\partial \alpha_{i-1}}{\partial x_{1}} \psi_{1,1} x_{2}, \ldots,-\frac{\partial \alpha_{i-1}}{\partial x_{i-1}} \psi_{1, i-1} x_{i},\right. \\
\left.\psi_{i}^{T},-\frac{\partial \alpha_{i-1}}{\partial x_{1}} \psi_{1}^{T}, \ldots,-\frac{\partial \alpha_{i-1}}{\partial x_{i-1}} \psi_{i-1}^{T}\right]^{T}
\end{gathered}
$$

Then (20) becomes

$$
\begin{aligned}
z_{i} \dot{z}_{i} \leq & {\left[g_{i}\left(\bar{x}_{i}\right) z_{i} z_{i+1}+z_{i} g_{i}\left(\bar{x}_{i}\right) \alpha_{i}+\Theta_{i}^{T} \Phi_{i}\left(\bar{x}_{i}\right) z_{i}\right.} \\
& \left.+\beta_{i}\right]+\lambda_{i}^{*}\left|z_{i}\right| \bar{\phi}_{i}\left(\bar{x}_{i}\right)
\end{aligned}
$$

Note that (5) and (7) (11), similar to Step 1, we can get

$$
\begin{aligned}
\dot{V}_{i} \leq & -\left(c_{i}-\frac{1}{4}\right) z_{i}^{2}-\frac{1}{2} \sigma_{\theta i}\left|\tilde{\Theta}_{i}\right|^{2}-\frac{1}{2} \sigma_{\lambda i}\left|\tilde{\lambda}_{i}^{*}\right|^{2} \\
& +\left(g_{i} N\left(\kappa_{i}\right)+1\right) \dot{\kappa}_{i}+g_{i} z_{i+1}^{2}+\delta_{i} \\
\leq & -c_{i i} V_{i}+\left(g_{i} N\left(\kappa_{i}\right)+1\right) \dot{\kappa}_{i}+\delta_{i}+g_{i}^{2} z_{i+1}^{2}
\end{aligned}
$$

Similar to Step 1, then

$$
\begin{aligned}
& V_{i}(t) \leq \rho_{i}+V_{i}(0)+e^{-c_{11} t} \int_{0}^{t} g_{i} N\left(\kappa_{i}\right) \dot{\kappa}_{i} e^{c_{i i} \tau} d \tau \\
& \quad+e^{-c_{11} t} \int_{0}^{t} \dot{\kappa}_{i} e^{c_{11} \tau} d \tau+e^{-c_{11} t} \int_{0}^{t} g_{i}^{2} z_{i+1}^{2} e^{c_{11} \tau} d \tau
\end{aligned}
$$

Similar to the analysis in Step 1 , if $z_{i+1}$ is regulated as bounded in the next step, then the boundedness of $z_{i}$ can be readily concluded.

Step $\boldsymbol{n}$ : Define $z_{n}=x_{n}-\alpha_{n-1}$. By setting $i=n$ in (5) and (7) (11), and $z_{n+1}=0$, then

$$
\begin{aligned}
\dot{V}_{n} \leq & -c_{n} z_{n}^{2}-\frac{1}{2} \sigma_{\theta n}\left|\tilde{\Theta}_{n}\right|^{2}-\frac{1}{2} \sigma_{\lambda n}\left|\tilde{\lambda}_{n}^{*}\right|^{2} \\
& +\left(g_{n} N\left(\kappa_{n}\right)+1\right) \dot{\kappa}_{n}+\delta_{n} \\
\leq & -c_{n n} V_{n}+\left(g_{n} N\left(\kappa_{n}\right)+1\right) \dot{\kappa}_{n}+\delta_{n}
\end{aligned}
$$

Let $\rho_{n}=\delta_{n} / c_{n n}$, similarly,

$$
\begin{aligned}
V_{n}(t) \leq & \rho_{n}+V_{n}(0)+e^{-c_{11} t} \int_{0}^{t} g_{n} N\left(\kappa_{n}\right) \dot{\kappa}_{n} e^{c_{n n} \tau} d \tau \\
& +e^{-c_{11} t} \int_{0}^{t} \dot{\kappa}_{n} e^{c_{11} \tau} d \tau
\end{aligned}
$$

By choosing $c_{0}=\rho_{n}+V_{n}(0)$, According to Lemma 1 and Remark 2, it can be concluded that $V_{n}(t), \kappa_{n}(t)$, hence $\alpha_{n}, z_{n}(t), \hat{\Theta}_{n}$ and $\hat{\lambda}_{n}^{*}$ are GUUB. From the boundedness of $z_{n}(t)$, the boundedness of the extra term at Step $(n-1)$ is obtained. Applying Lemma 1 for $(n-1)$ times backward, it can be seen from the above iterative design procedures that $V_{i}, \alpha_{i}, z_{i}, \hat{\Theta}_{i}, \hat{\lambda}_{i}^{*}$ and hence $x_{i}(t)$ are GUUB, $i=1, \cdots, n$. Since $y(t)=$ $x_{1}(t)=z_{1}(t)+y_{d}(t)$, from the definition of $V_{1}$ and (17), the property (12) can be easily obtained. In addition, by appropriately choosing the design constants the regulation of the tracking error $z_{1}$ to any prescribed accuracy can be achieved while keeping the boundedness of all signals and states involved.

The proof is complete.

\section{ILLUSTRATIVE EXAMPLE}

\subsection{A Mathematical Example}

Consider the following uncertain system,

$$
\left\{\begin{aligned}
\dot{x}_{1}(t)= & \left(1+2 x_{1}^{2}\right) x_{2}+2 x_{1}^{2}+0.6 x_{2} \sin \left(x_{2}\right) \\
\dot{x}_{2}(t)= & \left(3+\cos \left(x_{1} x_{2}\right)\right) u+0.5 x_{2} \sin \left(x_{2}\right) \\
& \quad+0.5\left(x_{1}^{2}+x_{2}^{2}\right) \sin (t) \\
y(t)= & x_{1}(t)
\end{aligned}\right.
$$

where $g_{1}\left(x_{1}\right)=1+2 x_{1}^{2}, g_{2}(x)=3+\cos \left(x_{1} x_{2}\right)$, $\psi_{1}\left(x_{1}\right)=x_{1}^{2}, \psi_{2}(x)=x_{2} \sin \left(x_{2}\right), \Delta_{2}=$ $0.5\left(x_{1}^{2}+x_{2}^{2}\right) \sin (t), \Delta_{1}=0.6 \sin \left(x_{2}\right), \theta_{1}=$ $2, \theta_{2}=0.5$. If choose $\lambda_{1}=0.6, \lambda_{2}=0.5$, $\phi_{1}=1, \phi_{2}=\left(x_{1}^{2}+x_{2}^{2}\right)$, then Assumption 3 holds. The reference signal is chosen as in (Ge, et al., 2003): $y_{d}=0.5(\sin (t)+\sin (0.5 t))$. Consequently, the robust adaptive tracking controller can be achieved as in (9)-(11) and (6), $i=1,2$, $n=2$. Where $\lambda_{1}^{*}=\lambda_{1}, \lambda_{2}^{*}=\max \left\{\lambda_{1}, \lambda_{2}\right\}$, $\beta_{1}=-\dot{y}_{d}, \beta_{2}=-\frac{\partial \alpha_{1}}{\partial \hat{\theta}_{1}} \dot{\hat{\theta}}_{1}-\frac{\partial \alpha_{1}}{\partial \hat{\lambda}_{1}} \dot{\hat{\lambda}}_{1}-\sum_{j=0}^{1} \frac{\partial \alpha_{1}}{\partial y_{d}^{(j)}} y_{d}^{(j+1)}-$ $\frac{\partial \alpha_{i-1}}{\partial \kappa_{1}} \dot{\kappa}_{1}, \bar{\phi}_{1}=\phi_{1}, \bar{\phi}_{2}(x)=\phi_{2}+\left|\frac{\partial \alpha_{1}}{\partial x_{1}}\right| \phi_{1}, \Theta_{1}=\theta_{1}$, $\Theta_{2}=\left[b_{1}, \theta_{2}^{T}, \theta_{1}^{T}\right]^{T}, \psi_{1,1}\left(x_{1}\right)=0.5+x_{1}^{2}, b_{1}=2$, $\Phi_{1}=\psi_{1}, \Phi_{2}=\left[-\frac{\partial \alpha_{1}}{\partial x_{1}} \psi_{1,1} x_{2}, \psi_{2}^{T},-\frac{\partial \alpha_{1}}{\partial x_{1}} \psi_{1}^{T}\right]^{T}$.

The design constants are chosen as $c_{1}=1, c_{2}=20$ , $\sigma_{\theta 1}=0.5, \sigma_{\theta 2}=2, \sigma_{\lambda 1}=\sigma_{\lambda 2}=2, \gamma_{1}=\gamma_{2}=$ $0.05, \Gamma_{1}=\Gamma_{2}=0.5, \varepsilon_{1}=\varepsilon_{2}=0.01, \quad \sigma_{b 1}=$ 0.5. The following initial conditions are adopted as $x(0)=[-0.5,0]^{T}, \theta_{1}^{0}=0.5, \theta_{2}^{0}=0.1$, $\hat{\theta}_{1}(0)=\hat{\theta}_{2}(0)=\hat{\lambda}_{1}(0)=\hat{\lambda}_{2}(0)=\hat{b}_{1}(0)=0$.

Simulation results in Figs.1-3 show the effectiveness of the presented controller for system (31). Fig. 1 shows that the output tracks the reference signal $y_{d}$ perfectly after 10 seconds just like (Ge et al., 2003). Figs. 2-3 illustrate the boundedness of some signals and variables respectively.

\subsection{Practical Example-Linear Track-keeping Control of Ships}

To illustrate the practicability of the interesting systems in this paper, a practical example on ship 


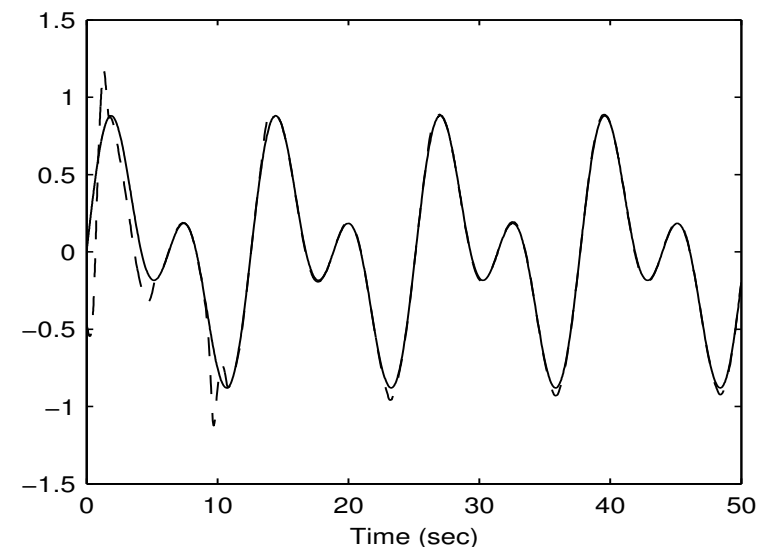

Fig. 1. Output $y(t)\left("-\right.$-") and reference $y_{d}("-")$.

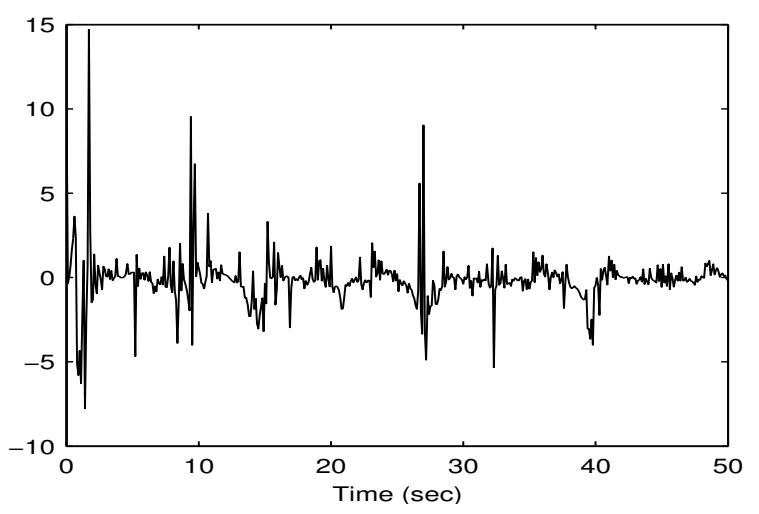

Fig. 2. Control input $u(t)$.
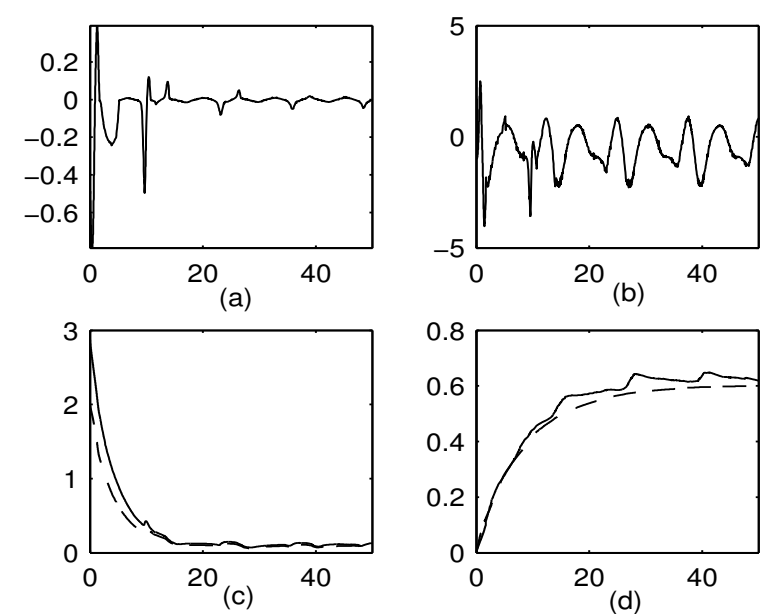

Fig. 3. (a) tracking error $z_{1}$. (b) state $x_{2}$. (c) Norms $\left\|\hat{\Theta}_{1}\right\|("--")$ and $\left\|\hat{\Theta}_{2}\right\|("-")$. (d) Estimates $\hat{\lambda}_{1}("--")$ and $\hat{\lambda}_{2}("-")$.

linear track-keeping control design is presented. The following nonlinear ship straight-line motion equations ( $\mathrm{Li}$ and Yang, 2004) is

$$
\left\{\begin{array}{l}
\dot{y}=U \sin (\psi) \\
\dot{\psi}=r \\
\dot{r}=f_{2}(r)+b(t) u+w
\end{array}\right.
$$

where $y, \psi, r$ and $U$ denote the sway displacement (cross-track error), heading angle, yaw rate and cruise speed respectively. $f_{2}$ depicts the uncertain dynamics, $b=K / T$. In this paper, we assume that $U, T, K$ and $\alpha$ are unknown but constant. $u$ denotes rudder angle input; $w$ denotes equivalent external perturbations induced by current, wave and wind.

Remark 3: In practice, the high gain $b=K / T$ is slowly time-varying, i.e., $b=g(t)$, and the sign of $b$ is determined via the ship test or the trial-anderror way. So in this sense, our proposed algorithm can ease the controller design in practice.

With the coordinate transformation

$$
\left\{\begin{array}{l}
x_{1}=\psi+\arcsin \left(\frac{k y}{\sqrt{1+(k y)^{2}}}\right) \\
x_{2}=r
\end{array}\right.
$$

We can get the following systems of the form (1)

$$
\left\{\begin{array}{l}
\dot{x}_{1}=f_{1}(\cdot)+x_{2}+\Delta_{1} \\
\dot{x}_{2}=f_{2}(x)+g(t) u+\Delta_{2} \\
\eta=x_{1}
\end{array}\right.
$$

where $f_{1}=k U \sin (\psi) /\left(1+(k y)^{2}\right), \Delta_{1}=0, \Delta_{2}=$ $w, \eta=x_{1}$ is the output.

Now, the regulation of (32) becomes that of (34)( refer to (Li and Yang, 2004) for details).

With our proposed algorithm $(6) \sim(11)$, the simulation results are demonstrated by Fig.4 and Fig.5 as follows.

Remark 4: The ship roll stabilization system, which is a typical 2 nd order dynamic system

$$
\begin{aligned}
& \left(I_{x x}+J_{x x}\right) \ddot{\varphi}+N \dot{\varphi}+W \dot{\varphi}|\dot{\varphi}| \\
& \quad+D h \varphi\left(1-\left(\varphi / \varphi_{v}\right)^{2}\right)=F_{C}+F_{W},
\end{aligned}
$$

controlled by fin with actuator, can be transformed into the form of (1) as

$$
\left\{\begin{array}{l}
\dot{x}_{1}=x_{2} \\
\dot{x}_{2}=f_{2}\left(\bar{x}_{2}\right)+g_{2}\left(\bar{x}_{2}\right) x_{3}+\Delta_{2} \\
\dot{x}_{3}=f_{3}(x)+g_{3}(x) u+\Delta_{3}
\end{array}\right.
$$

refer to (Yang et al., 2004) (Yang et al., 2003) and (Yang and Zhou, 2005) for details.

\section{CONCLUSION}

In this paper, a novel robust adaptive tracking controller has been developed for a large class of strict feedback uncertain nonlinear perturbed systems without a priori knowledge of the signs of the unknown virtual control coefficients. It proved that the proposed control algorithm is able to guarantee GUUB of all the signals. In addition, the output of the system has been proven to converge to a small neighborhood of the origin. Numerical simulation results are presented to validate the effectiveness of the proposed approach. 

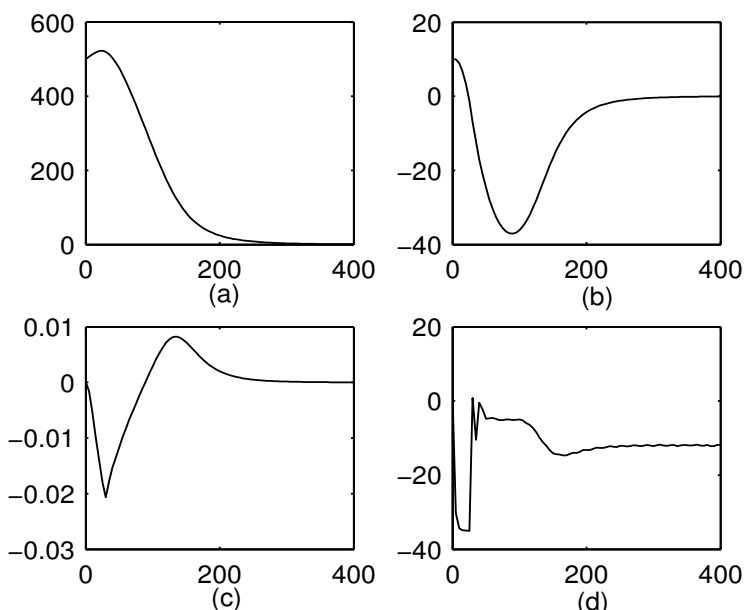

Fig. 4. (a) Cross-track error $y(\mathrm{~m})$. (b) Heading error $\psi(\mathrm{deg})$. (c) Yaw rate $r(\mathrm{rad} / \mathrm{s})$. (d) Rudder input $u(\operatorname{deg})$.

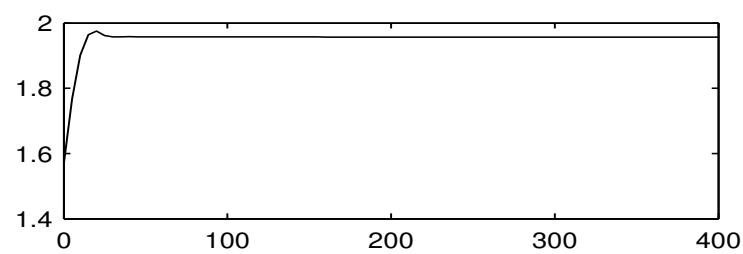

(a)

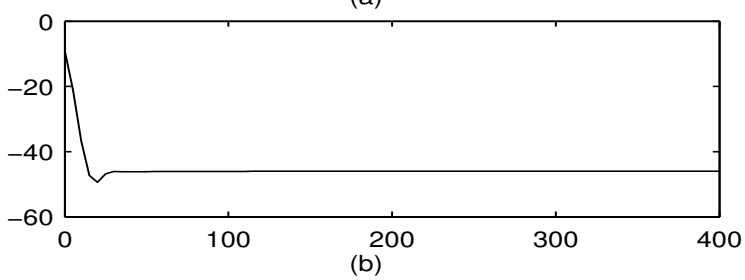

Fig. 5. (a) Adaptation parameter $\kappa$. (b) Nussbaum function $N(\kappa)$.

In a word, this paper enlarges the class of strictfeedback uncertain nonlinear systems with unknown virtual control gain functions under perturbation for which global robust adaptive tracking control can be designed.

\section{REFERENCES}

\section{REFERENCES}

Ge, S. S. and J. Wang (2002). Robust adaptive neural control for a class of perturbed strict feedback nonlinear systems. IEEE Trans. on Neural Networks, 13(6), 1409-1419.

Ge, S. S., F. Hong and T. H. Lee (2003). Adaptive neural control of nonlinear time-delay systems with unknown virtual control coefficients. IEEE Trans. on Systems, Man and Cybernetics-Part B: Cybernetics, 1, 1-18.

Isidori, A. (1995). Nonliear Control Systems, 2nd ed. Springer-Verlay. Berlin.

Kanellakopoulos, I. (1993). assive adaptive control of nonlinear systems. Int. J. of Adaptive Control and Signal Processing, 7, 339-352.
Kanellakopoulos, I., P. V. Kokotovic and A. S. Morse (1991). Systematic design of adaptive controllers for feedback linearizable systems. IEEE Trans. on Automatic Control, 36, 1241-1253.

Krstic, M., I. Kanellakopoulos and P. V. Kokotovic (1992). Adaptive nonlinear control without oveparametrization. Systems and Control Letter, 19, 177-185.

Krstic, M., I. Kanellakopoulos and P. V. Kokotovic (1995). Nonlinear and Adaptive Control Design. Wiley. New York.

Li, T. S. and Y. S. Yang (2004). Robust dissipative designs for straight-line tracking control of underactuated ships. Proceedings of WCICA'04, Hangzhou, China. pp. 548-552.

Marino, R. and P. Toper (1993). Global adaptive output-feedback control of nonlinear systems, part i: Linear parameterization; part ii: nonlinear parameterization. IEEE Trans. on Automatic Control, 38, 17-49.

Nussbaum, R. D. (1983). Some remarks on the conjecture in parameter adaptive control. Systems and Control Letter, 3, 243-246.

Ryan, E. P. (1991). A universal adaptive stabilizer for a class of nonlinear systems. Systems and Control Letter, 16, 209-218.

Sastry, S. S. and A. Isidori (1989). Adaptive control of linearization systems. IEEE Trans. on Automatic Control, 34, 1123-1131.

Yang, Y. S. and C. J. Zhou (2005). Adaptive fuzzy $h_{\infty}$ stabilization for strict-feedback canonical nonlinear systems via backstepping and small-gain approach. IEEE Transactions on Fuzzy Systems, in press.

Yang, Y. S., C. J. Zhou and T. S. Li (2003). Small gain method for adaptive robust fuzzy control of a class of nonlinear systems. Proceedings of the 2003 IEEE International Symposium on Intelligent Control, Houston, Texas, pp. 4045.

Yang, Y. S., G. Feng and J. S. Ren (2004). A combined backstepping and small-gain approach to robust adaptive fuzzy control for strictfeedback nonlinear systems. IEEE Transactions on Systems, Man, and Cybernetics Part A:Systems and Humans, pp. 406-420.

Ye, X. D. and J. P. Jiang (1998). Adaptive nonlinear design without a priori knowledge of control directions. IEEE Trans. on Automatic Control, 43(11), 1617-1621. 